\title{
Eficacia del Índice de Mannheim en pacientes con peritonitis secundaria de un hospital de Huancayo, Perú
}

\begin{abstract}
Efficacy of the Manheim's index in patients with secondary peritonitis in a hospital in Huancayo, Peru
\end{abstract}
Carlos Sedano ${ }^{1, a}$, Leo Lizano ${ }^{1, a}$, Javier Balbin ${ }^{1, b, c}$, Ivan Condor ${ }^{1, a}$, Joel Atencio ${ }^{1, d}$, Carlos Villalba ${ }^{1, d}$

\section{RESUMEN}

Objetivos: Determinar la eficacia del Índice de Mannheim como predictor de mortalidad en pacientes con peritonitis secundaria. Material y métodos: Estudio observacional retrospectivo, tipo prueba diagnóstica. realizado con los datos de las historias clínicas. A través de un muestreo no probabilístico se analizó 294 pacientes: 40 fallecidos y 254 sobrevivientes postoperados de peritonitis secundaria del Servicio de Cirugía General del Hospital "Daniel Alcides Carrión" de Huancayo entre los años 2015 y 2017; se calculó dicho tamaño muestral con el uso del estadístico EPIDAT 4.1 para pruebas diagnósticas. Se determinó: Sensibilidad, especificidad, valor predictivo positivo, valor predictivo negativo, razón de verosimilitud positivo, razón de verosimilitud negativa, curva ROC y el área bajo la curva ROC (ABC). Resultados: El Índice de Mannheim con el punto de corte utilizado según el trabajo original de $\geq 26$ mostró una sensibilidad de 0,85 , especificidad 0,972 , valor predictivo positivo de 0,829 , valor predictivo negativo 0,976. El punto de corte optimo con una sensibilidad de 0,975 se obtuvo en el punto de corte $\geq 21$. El área bajo la curva ROC fue 0,976. Conclusiones: El Índice de Mannheim demostró ser un buen predictor de mortalidad, estableciéndose que el mejor punto de corte es $\geq 21$ para aumentar la sensibilidad al $97,5 \%$ a fin de evitar la pérdida de casos detectados por el Índice.

PALABRAS CLAVE: Peritonitis, Curva ROC, predicción. (Fuente: MeSH NLM).

\section{SUMMARY}

Objectives: To determine the efficacy of the Manheim's index in predicting mortality in patients with secondary peritonitis. Methods: A retrospective diagnostic like study was carried-out with data extracted from clinical charts. A non-probabilistic sampling of 294 patients of whom 40 had died and 254 were discharged alive after being surgically intervened from secondary peritonitis in the General Surgery service at Hospital Daniel Alcides Carrion in Huancayo was calculated using EPIDAT 4.1. Sensitivity, specificity, predictive values, likelihood ratios and ROC curves and area under the ROC were calculated. Results: A Manheim's index of $\geq 26$ showed a sensitivity of 0.85 , specificity of 0.972 , positive predictive value of 0.829 and negative predictive value of 0.976 . The optimal threshold was $\geq 21$ attaining a sensitivity of 0.975 . The area under the ROC curve was 0.976 . Conclusions: Manheim's index is a good predictor of mortality among patients with secondary peritonitis, the optimal threshold was $\geq 21$.

KEYWORDS: Peritonitis, ROC Curve, forecasting. (Source: MeSH NLM).

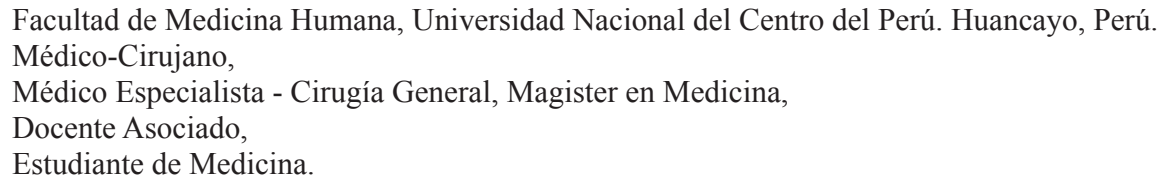




\section{INTRODUCCIÓN}

La peritonitis generalizada secundaria todavía es una de las patologías más letales, es por ello importante su pronta identificación por el personal médico, recordando que es fundamental que la población también tenga conocimientos y que la comunidad médica tenga sistemas de predicción de mortalidad. En la época antes del tratamiento quirúrgico la clínica de esta patología seguía su curso natural llegando a tener una mortalidad del $88 \%$ (1). En las prioridades del Ministerio de Salud del Perú para los años 2015 y 2021 establecidos por el Instituto Nacional de Salud, está como octava prioridad las afecciones del apéndice y de los intestinos patologías muy relacionadas con la peritonitis secundaria (2).

Los pacientes sometidos a cirugía por peritonitis secundaria tienen por lo general complicaciones que todo cirujano debe enfrentar y que son inherentes a dicho procedimiento y que se intensifica si el paciente tiene otros factores asociados; es así que un pronóstico favorable es el uso de una antibioterapia adecuada y un control del origen lo cual nos reduce la mortalidad de esta patología a 5\%-6\% (3).

El Índice de Mannheim de Peritonitis (IMP) desarrollado con 1253 pacientes con peritonitis en los que de 20 ítems inicialmente, 8 demostraron ser predictores de mortalidad $(4,5)$. Los factores que incluye este índice son: Edad, sexo, falla orgánica, presencia de malignidad, origen, extensión de la peritonitis, características del fluido peritoneal, siendo el rango de 0 a 47 puntos. El índice de Mannheim en comparación con el sistema de clasificación APACHE II es de fácil aplicación al ofrecer una estimación personalizada sobre el riesgo de mortalidad, siendo de rápido acceso y por lo general es un sistema predictivo práctico en patologías como la peritonitis secundaria (5).

El estudio tuvo como objetivo determinar la eficacia del Índice de Mannheim como pronóstico de mortalidad, en pacientes con peritonitis secundaria.

\section{MATERIAL Y MÉTODOS}

Estudio observacional, retrospectivo, tipo prueba diagnóstica.

Se revisaron las historias clínicas de los pacientes con diagnóstico de peritonitis secundaria del servicio de Cirugía general del Hospital "Daniel Alcides
Carrión" de Huancayo del periodo 2015 al 2017, y que cumplían con los criterios de selección.

Para hallar el tamaño de la muestra se utilizó el programa estadístico epidemiológico EPIDAT 4.1 para pruebas diagnósticas, teniendo en cuenta el estudio de Barrera (6), con una sensibilidad de 96\%, especificidad $80 \%$, nivel de confianza del $95 \%$, el resultado fue 294 pacientes, siendo 40 fallecidos y 254 sobrevivientes con una precisión al 95\%. El muestreo fue no probabilístico, se seleccionaron los pacientes que cumplían con los siguientes criterios de selección:

\section{Criterios de inclusión:}

Pacientes postoperados que cumplían con la definición de peritonitis secundaria establecido para el estudio, hospitalizados en el Servicio de Cirugía durante los años 2015 - 2017.

Pacientes que tengan historias clínicas completas en el almacenamiento del archivo de historias clínicas del hospital.

\section{Criterios de exclusión:}

Pacientes con peritonitis secundaria de causa ginecológica o urológica.

Pacientes que estén cursando con una peritonitis terciara definida como luego de la presencia de una peritonitis secundaria o después de 3 reintervenciones con laparotomías ya planeadas.

Se definió "peritonitis secundaria" consecutiva a una contaminación de la cavidad peritoneal por perforación o inflamación e infección grave de un órgano intraabdominal siendo las causas incluidas para este estudio las siguientes: apendicitis aguda, perforación de víscera hueca, obstrucción intestinal (perforación), colecistitis aguda, absceso hepático (rotura), traumatismo abdominal (perforación), absceso residual intraabdominal apendicular (rotura), quiste hidatídico (rotura), trombosis mesentérica (necrosis), neoplasias con metástasis al hígado (abscedadas), perforación yeyunal por bezoar, cirrosis hepática con peritonitis secundaria, fistula biliar, hematoma retroperitoneal, duodenitis crónica por estrongiloidosis (perforación), tumoración abdominal (obstrucción y perforación), ulcera gastroduodenal perforado, abscesos pancreáticos, dehiscencia de una anastomosis intestinal, perforación de un divertículo sigmoideo, cuerpos extraños ingeridos o naturales (cálculos biliares), derrame de líquidos naturales: 
Tabla 1. Índice de Pronóstico de mortalidad de peritonitis de Mannheim.

\begin{tabular}{lcccc}
\hline Factor & Positivo & Puntaje & Negativo & Puntaje \\
\hline 1. Edad & Mayor de 50 años & 5 & Menor de 50 años & 0 \\
2. Sexo & Femenino & 5 & Masculino & 0 \\
3. Falla Orgánica & Presente & 7 & Ausente & 0 \\
4. Cáncer & Presente & 4 & Ausente & 0 \\
5. Tiempo de evolución & $\geq 24$ Horas & 4 & $<24$ Horas & 0 \\
6. Origen & No colónico & 4 & Colónico & 0 \\
7. Extensión de la peritonitis & Generalizada & 4 & Localizada & 0 \\
8. Características del Fluido & Fecaloide & 12 & Claro & 0 \\
\hline
\end{tabular}

Tabla 2. Características generales de los pacientes con peritonitis secundaria.

\begin{tabular}{|c|c|c|}
\hline & $\mathbf{n}$ & $\%$ \\
\hline \multicolumn{3}{|l|}{ Género } \\
\hline Masculino & 181 & 61,6 \\
\hline Femenino & 113 & 38,4 \\
\hline Total & 294 & 100,0 \\
\hline \multicolumn{3}{|l|}{ Etiología } \\
\hline Apendicitis aguda Complicada & 217 & 73,8 \\
\hline Perforación de víscera hueca & 22 & 7,5 \\
\hline Obstrucción Intestinal (perforación) & 18 & 6,1 \\
\hline Colecistitis aguda complicada & 12 & 4,1 \\
\hline Absceso hepático (Rotura) & 3 & 1,0 \\
\hline Traumatismo abdominal (Perforación) & 3 & 1,0 \\
\hline Absceso residual intraabdominal apendicular (Rotura) & 3 & 1,0 \\
\hline Quiste hidatídico (Rotura) & 2 & 0,7 \\
\hline Trombosis mesentérica (Necrosis) & 2 & 0,7 \\
\hline Neoplasias con metástasis al hígado (Abscedadas) & 2 & 0,7 \\
\hline Perforación yeyunal por bezoar & 2 & 0,7 \\
\hline Cirrosis hepática con Peritonitis secundaria & 1 & 0,3 \\
\hline Fistula biliar & 1 & 0,3 \\
\hline Hematoma retroperitoneal & 1 & 0,3 \\
\hline Duodenitis crónica por Estrongiloidosis (Perforación) & 1 & 0,3 \\
\hline Tumoración abdominal (Obstrucción y perforación) & 1 & 0,3 \\
\hline Otros & 3 & 1,0 \\
\hline Total & 294 & 100,0 \\
\hline \multicolumn{3}{|l|}{ Mortalidad } \\
\hline Sobrevivientes & 254 & 86,4 \\
\hline Fallecidos & 40 & 13,6 \\
\hline Total & 294 & 100,0 \\
\hline
\end{tabular}


bilis, orina y sangre; Isquemia, infarto de epiplón y vólvulos con perforación $(7,8)$.

El índice de Mannheim es una herramienta de pronóstico de mortalidad que cuenta con 8 parámetros clínicos y quirúrgicos (tabla 1). Fue validado y publicado en 1987 en Alemania por los médicos Wacha L, Linder $\mathrm{M}$ et al., donde establecieron el punto de corte 26; cada factor tiene un puntaje si es positivo o negativo siendo el rango de puntuación final entre 0 a 47 puntos luego de la suma de cada factor.
La valoración final es si tiene un puntaje $<26$ bajo de riesgo de mortalidad y $>26$ riesgo alto de mortalidad $(9,11)$.

En primera instancia se utilizó estadística descriptiva para determinar frecuencias y medidas de tendencia central y luego se determinó sensibilidad, especificidad, valor predictivo positivo, valor predictivo negativo, razón de verosimilitud positivo, razón de verosimilitud negativa, curva ROC y área bajo la curva ROC (ABC); para ello se usaron los

Tabla 3. Características de los pacientes fallecidos con peritonitis secundaria.

\begin{tabular}{lcc}
\hline & $\mathbf{n}$ & $\mathbf{\%}$ \\
\hline Sexo & 21 & 52,5 \\
Masculino & 19 & 47,5 \\
Femenino & 40 & 100,0 \\
Total & & \\
Etiología & 10 & 25,0 \\
Perforación de víscera hueca & 9 & 22,5 \\
Obstrucción Intestinal perforación & 5 & 12,5 \\
Apendicitis aguda complicada & 4 & 10,0 \\
Colecistitis aguda complicada & 2 & 5,0 \\
Absceso hepático (Rotura) & 2 & 5,0 \\
Trombosis mesentérica (Necrosis) & 2 & 5,0 \\
Neoplasias metástasis al hígado (Abscedadas) & 2 & 5,0 \\
Perforación yeyunal por bezoar & 1 & 2,5 \\
Quiste hidatídico (Rotura) & 1 & 2,5 \\
Hematoma retroperitoneal & 1 & 2,5 \\
Duodenitis crónica por estrongiloidosis (Perforación) & 1 & 2,5 \\
Tumoración abdominal (Obstrucción y perforación) & 40 & 100,0 \\
Total & & \\
\hline
\end{tabular}

Tabla 4. Tasa de mortalidad según puntos de corte del Índice de Mannheim.

\begin{tabular}{lccccc}
\hline Índice de & \multicolumn{2}{c}{ Sobrevivientes } & \multicolumn{2}{c}{ Fallecidos } & \multirow{2}{*}{ Tasa de mortalidad (\%) * } \\
\cline { 2 - 5 } Mannheim & $\mathbf{n}$ & $\mathbf{\%}$ & $\mathbf{n}$ & $\mathbf{\%}$ & \\
\hline$<21$ & 222 & $87,4 \%$ & 1 & $2,5 \%$ & 0,45 \\
$21-29$ & 29 & $11,4 \%$ & 10 & $25,0 \%$ & 25,64 \\
$>29$ & 3 & $1,2 \%$ & 29 & $72,5 \%$ & 90,63 \\
\hline
\end{tabular}

* Tasa de mortalidad: Porcentaje de fallecidos respecto al total de pacientes expuestos a los diferentes puntos de corte (filas). 
Tabla 5. Medidas de eficacia del Índice de Mannheim para el punto de corte $\geq 26$.

\begin{tabular}{lccc}
\hline Medidas métricas de evaluación diagnostica & Valor & \multicolumn{2}{c}{ IC (95\%) } \\
\hline Prevalencia (\%) & 13,61 & 9,52 & 17,69 \\
Sensibilidad: S (\%) & 85,00 & 72,68 & 97,32 \\
Especificidad: E (\%) & 97,24 & 95,03 & 99,45 \\
Valor predictivo+: VPP (\%) & 82,93 & 70,19 & 95,66 \\
Valor predictivo-: VPN (\%) & 97,63 & 95,56 & 99,70 \\
Índice de Youden: IY & 0,82 & 0,71 & 0,93 \\
Área Bajo la Curva ROC (ABC) & 0,976 & 0,951 & 0,990 \\
\hline
\end{tabular}

programas estadísticos EPIDAT 3.1, EPIDAT 4.1, SPSS 23 y MedCalc.

El estudio contó con la aprobación del comité de investigación de la Facultad de Medicina Humana de la Universidad Nacional del Centro del Perú, así mismo por el comité de ética, docencia e investigación del Hospital "Daniel Alcides Carrión” de Huancayo.

\section{RESULTADOS}

Se revisaron las historias clínicas de 294 pacientes; la mortalidad fue $13,6 \%$. Las características generales de los pacientes con peritonitis secundaria se pueden ver en la tabla 2 ; el $61,6 \%$ fue de sexo femenino, la edad media fue 42,21 $\pm 17,39$ años, la mediana 39 , con un rango Inter cuartil (IRQ) 16; las etiologías más frecuentes de peritonitis secundaria fueron: Apendicitis aguda complicada $73,8 \%$, perforación de víscera hueca $7,5 \%$, obstrucción intestinal perforado $6,1 \%$, colecistitis aguda complicada $4,1 \%$ y rotura de absceso hepático, traumatismo abdominal con perforación y el absceso residual intraabdominal apendicular cada uno $1 \%$.

En la tabla 3 se muestran las características de los pacientes fallecidos; el 52,5\% era de sexo masculino. Las etiologías más frecuentes fueron: perforación de víscera hueca $25 \%$, obstrucción intestinal perforado $22,5 \%$, apendicitis aguda complicada $12,5 \%$, colecistitis aguda complicada $10 \%$ y rotura de absceso hepático, trombosis mesentérica (necrosis), neoplasias metastásicas al hígado (abscedadas) y perforación yeyunal por bezoar, cada uno $5 \%$. El promedio de edad de los pacientes fallecidos fue 63,40 años y en los supervivientes fue 38,77 años; el promedio del puntaje del Índice de Mannheim en los pacientes fallecidos fue 30,95 y en los sobrevivientes 13,61 .
En la tabla 4 se muestra la mortalidad según grupos de corte del estudio de Billing et al., (9), del Índice de Mannheim de 21 y 29. Para el punto de corte $\geq 26$, la sensibilidad fue $85 \%$, la especificidad $97,24 \%$, el valor predictivo positivo (VPP) $82,93 \%$, valor predictivo negativo (VPN) 97,63, razón de verosimilitud positiva 30,84 , razón de verosimilitud negativa 0,15 , la curva ROC y su consiguiente área bajo la curva 0,976 con un IC al 95\%:(0,951-0,990) (tabla 5 y gráfico 1$)$.

Finalmente, en la tabla 6 se presentan los puntos de corte según los puntajes del Índice de Mannheim siendo el punto de corte mas óptimo el mayor de 20 o $\geq 21$ con una sensibilidad del $97,5 \%$, especificidad $87,4 \%$, razón de verosimilitud positiva 7,74 y razón de verosimilitud negativa 0,029 .

\section{DISCUSIÓN}

En nuestro estudio en la categorización con los puntos de corte 21 y 29 del índice de Mannheim se determinó que los pacientes con un puntaje $<21$ tenían una tasa de mortalidad de $0,45 \%$, de $21-293,85 \%$ y $>29$ fue $90,63 \%$. Respecto a estas tasas de mortalidad según Batra et al., en la India difieren identificando que para los puntajes $\leq 20$ era $0 \%$, de $21-293,85 \%$ y $\geq 30$ fue $20 \%$ siendo el punto de corte de la curva ROC 26 (10).

En nuestra investigación se identificó que el punto de corte óptimo fue $\geq 21$ con una sensibilidad de $97,5 \%$ y especificidad 87,4\%. En 1994 se publicó el estudio más grande realizado después de la publicación original en 1987 por Linder et al. (11), en dicho estudio se examinaron a 2003 pacientes en 7 centros diferentes de varios países de Europa estableciendo el punto de corte 26 ; la sensibilidad fue $86 \%$ y la especificidad $74 \%$, variando el punto de corte según el centro hospitalario donde se aplicaba, en algunos era 21 y en otros 29 . Una 


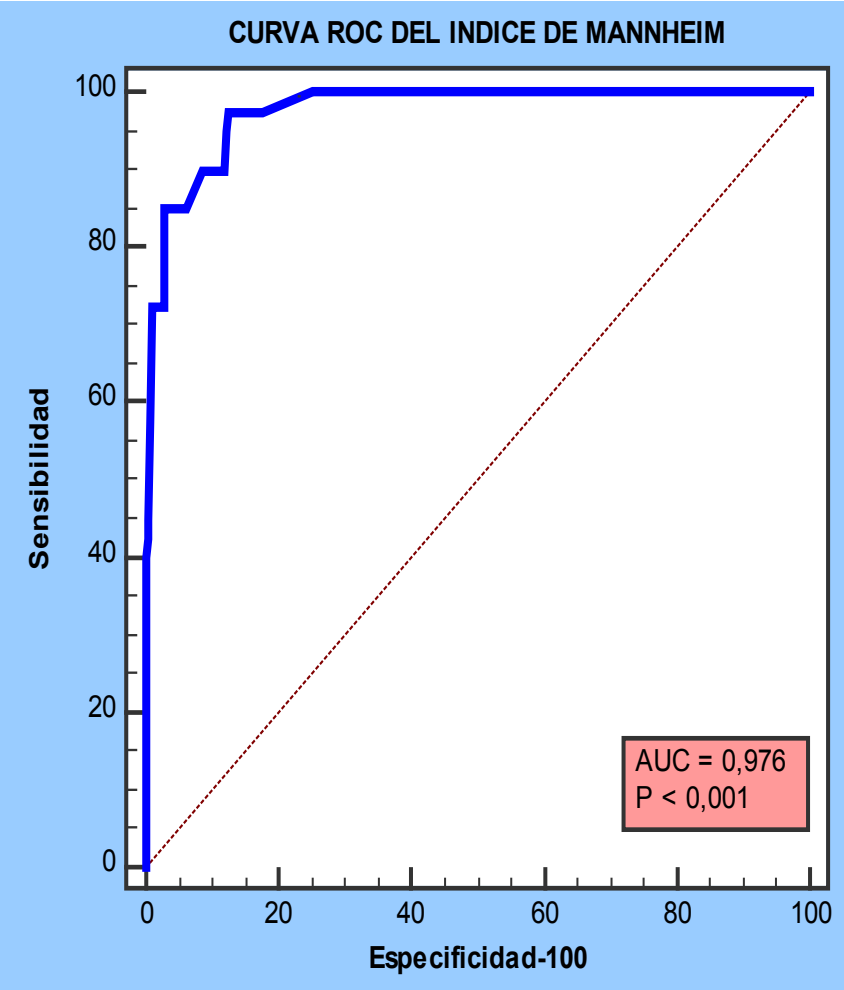

Gráfico 1. Curva ROC del Índice de Mannheim, con punto de corte $\geq 26$

Tabla 6. Puntos de corte de las medidas métricas de eficacia del Índice de Mannheim como predictor de mortalidad.

\begin{tabular}{ccccccc}
\hline Puntaje & Sensibilidad & IC del 95 \% & Especificidad & IC del 95 \% & + RV* & -RV* \\
\hline$\geq 0$ & 100,00 & $91,2-100,0$ & 0,00 & $0,0-1,4$ & 1,00 & \\
$>18$ & 100,00 & $91,2-100,0$ & 74,80 & $69,0-80,0$ & 3,97 & 0,00 \\
$>19$ & 97,50 & $86,8-99,9$ & 82,28 & $77,0-86,8$ & 5,50 & 0,030 \\
$>\mathbf{2 0}$ & $\mathbf{9 7 , 5 0}$ & $\mathbf{8 6 , 8}-\mathbf{9 9 , 9}$ & $\mathbf{8 7 , 4 0}$ & $\mathbf{8 2 , 7 - 9 1 , 2}$ & $\mathbf{7 , 7 4}$ & $\mathbf{0 , 0 2 9}$ \\
$>21$ & 95,00 & $83,1-99,4$ & 87,80 & $83,1-91,6$ & 7,78 & 0,057 \\
$>22$ & 90,00 & $76,3-97,2$ & 88,19 & $83,6-91,9$ & 7,62 & 0,11 \\
$>23$ & 90,00 & $76,3-97,2$ & 91,34 & $87,2-94,5$ & 10,39 & 0,11 \\
$>24$ & 85,00 & $70,2-94,3$ & 93,70 & $90,0-96,4$ & 13,49 & 0,16 \\
$>\mathbf{2 5}$ & $\mathbf{8 5 , 0 0}$ & $\mathbf{7 0 , 2}-\mathbf{9 4 , 3}$ & $\mathbf{9 7 , 2 4}$ & $\mathbf{9 4 , 4 - 9 8 , 9}$ & $\mathbf{3 0 , 8 4}$ & $\mathbf{0 , 1 5}$ \\
$>27$ & 72,50 & $56,1-85,4$ & 97,24 & $94,4-98,9$ & 26,31 & 0,28 \\
$>29$ & 72,50 & $56,1-85,4$ & 98,82 & $96,6-99,8$ & 61,38 & 0,28 \\
$>30$ & 57,50 & $40,9-73,0$ & 99,21 & $97,2-99,9$ & 73,03 & 0,43 \\
$>31$ & 45,00 & $29,3-61,5$ & 99,61 & $97,8-100,0$ & 114,30 & 0,55 \\
$>32$ & 42,50 & $27,0-59,1$ & 99,61 & $97,8-100,0$ & 107,95 & 0,58 \\
$>33$ & 40,00 & $24,9-56,7$ & 100,00 & $98,6-100,0$ & & 0,60 \\
$>40$ & 0,00 & $0,0-8,8$ & 100,00 & $98,6-100,0$ & & 1,00 \\
\hline
\end{tabular}

*+RV: razón de verosimilitud positiva, *-RV: razón de verosimilitud negativa. 
explicación muy importante respecto a las variaciones era la presencia de la malignidad que en algunos era menos frecuente, otro era el aspecto del líquido peritoneal como el fecaloide que era muy bajo; además la frecuencia de mortalidad para los puntajes fueron: $<21$ del $2 \%-3 \%$, de 21 a $2922,5 \%$ y finalmente $>29$ fue $59,1 \%$ (9).

Así por su parte en el Departamento de Cirugía en la India del Hospital Sina de Teherán en un estudio prospectivo con 80 pacientes el mejor punto de corte fue 21 con una sensibilidad del $100 \%$ y especificidad $79 \%$, en cambio para el punto de corte de 29 la sensibilidad era $79 \%$ y la especificidad $96 \%$ (12). del mismo modo el punto de corte varía como refiere en un trabajo de investigación en la India del año 2016 donde proponen que es 27 siendo su sensibilidad 66,67\% y especificidad del $100 \%$ con una precisión del 94\% (13). en contraposición en un estudio retrospectivo realizado en polonia en el año 2015 con 168 pacientes determinaron la mortalidad para los puntajes que fueron: $<21$ de $1,75 \%$, de $21-29$ $28,13 \%$ y $>29$ fue $50 \%$, estableciéndose el punto de corte de 32 y un área bajo la curva ROC(ABC) de $81 \%$ (14).

En nuestro país en un trabajo realizado en los Hospitales Belén y Regional Docente de Trujillo entre los años 2004 y el 2009 identificaron que el punto de corte fue 24,5 (15). En Ecuador para un punto de corte de 26 se estimó que la sensibilidad era $72,73 \%$ y la especificidad de $85,83 \%$ con un VPP 32\% y VPN 97,17\%, además las razones de verosimilitud fueron $\mathrm{RV}+$ de 5,13 y RV- 0,32 (16).

Carrillo-Hidalgo (17), sostiene que en el Hospital Regional de Alta Especialidad de Veracruz en México donde se analizó a 496 pacientes con diagnóstico de peritonitis el punto de corte optimo fue 26 puntos para pronosticar mortalidad con un $100 \%$ de sensibilidad, 91\% de especificidad, con un área bajo la curva ROC de 0,984; además existiendo asociación significativa en pacientes con edad mayor de 50 años, falla orgánica, malignidad, liquido turbio $\mathrm{y}$ liquido fecaloide. Vásquez (18), en su estudio tipo evaluación de prueba diagnóstica, con 95 pacientes con diagnóstico de sepsis intraabdominal en el Servicio de Cirugía del Hospital de Trujillo, estableció para el Índice de Mannheim el punto de corte 26 , con una sensibilidad de $77,4 \%$, especificidad $24,2 \%$, VPP $65,8 \%$, VPN 36,4\%; así mismo, comparo con el Apache II determinando que la sensibilidad era $95,2 \%$, especificidad $21,2 \%$, VPP $69,4 \%$, VPN $70 \%$,concluyendo que el punto más alto de sensibilidad para el índice de Mannheim fue de 24,5 y para el Apache II 16,5 (18). Finalmente, en un estudio realizado en el
Hospital Regional de Lambayeque se determinó que el punto de corte fue 27 con una sensibilidad de $100 \%$, especificidad $67,44 \%$, VPP $41,67 \%$, VPN $100 \%$, RV+ $3,07 \%$, RV- 0 y el área bajo la curva ROC de 0,8523 (1).

Los autores del índice de Mannheim, ante tal situación refieren que es importante tener las consideraciones que podrían afectar el punto de corte, como el personal médico, instalaciones hospitalarias y el sistema de salud de cada país; esto se hará muy evidente en países como la India, Brasil y Perú donde exponen un punto de corte menor que podría ser 21, u otros extremos como en Polonia donde llegaba a 32, aunque claro en la mayoría de estudios se acercaba al punto de corte 26 .

Las limitaciones del estudio son, al ser un estudio retrospectivo se pudo haber perdido información de la evolución de los pacientes en el tiempo como puede ser en la Unidad de Cuidados Intensivos (UCI); y por otro lado un análisis multivariado estratificado en variables confusoras como edad, sexo, liquido peritoneal y malignidad nos hubieran arrojado un análisis más exacto en referencia al valor pronóstico de mortalidad del Índice de Mannheim.

En conclusión, se determinó que el Índice de Mannheim mostro ser un predictor bueno de mortalidad, determinándose que el óptimo punto de corte es $\geq 21$ con una sensibilidad de $97,5 \%$ y con una área bajo la curva de 0,976 lo que significa que la probabilidad de tener un diagnóstico correcto como predictor de mortalidad será en el $97,6 \%$ de las ocasiones.

\section{Declaración de financiamiento y de conflictos de interés:}

El estudio fue financiado por los propios investigadores, quienes declaran no tener conflictos de interés y que ninguno de los autores ni sus familiares inmediatos tienen un acuerdo financiero o afiliación importante con productos, servicios empleados o abordados en el artículo o cualquier otro posible sesgo potencial en contra de otro producto, servicio o institución pública o privada.

\section{Contribución de autoría:}

CS, LL, JB, IC y JA: participaron en la concepción y diseño del trabajo, análisis e interpretación de datos, redacción del manuscrito, revisión crítica del manuscrito, aprobación de su versión final, asesoría estadística y asesoría técnica o administrativa. CV: Recolección / obtención de resultados. 


\section{Correspondencia:}

Joel Atencio

Av. Manantial 4817 Sapallanga, Huancayo, Perú.

Correo electrónico: yoel_005@yahoo.com

Celular: 51927999363

\section{REFERENCIAS BIBLIOGRÁFICAS}

1. Tineo L. Validación del índice pronóstico de Mannheim para mortalidad en pacientes con peritonitis generalizada en el Hospital Regional de Lambayeque. Tesis para optar el Título de Médico Cirujano. Chiclayo, Perú: Universidad de San Martín de Porres; 2017. 31 pp.

2. Rodriguez J. Valor pronóstico de morbimortalidad del índice de Mannheim en peritonitis secundaria en el servicio de cirugía del Hospital de Baja Complejidad Vitarte durante el periodo 2014-2016. Tesis para optar el Título de Médico Cirujano. Lima; Perú: Universidad Ricardo Palma; 2017. 55 pp.

3. Schwartz S, Brunicardi F, Andersen D. Principios de cirugía. Ciudad de México: McGraw-Hill Interamericana; 2015. p. 135-160.

4. Fraccalvieri D, Biondo $\mathrm{S}$. Índices pronósticos de mortalidad posoperatoria en la peritonitis del colon izquierdo. Revista Cirugía-española. 2009; 86(5):272277.

5. Muralidhar VA, Madhu CP, Sudhir S, Madhu S. Efficacy of Mannheim Peritonitis Index (MPI) Score in patients with secondary peritonitis. J Clin Diagn Res. 2014; 8(12):30-35.

6. Barrera E, Rodriguez M, Borda G, Najar N. Valor predictivo de mortalidad del índice de peritonitis de Mannheim. Rev Gastroenterol Peru. 2010; 30(3):211215.

7. Soler R. Abdomen agudo no traumático. Ciudad de la Habana, Cuba: Editorial Ciencias médicas; 2004. p. 7-73.

8. Townsend CM, Beauchamp RD, Evers BM, Mattox Kl. Sabinston tratado de cirugía. Barcelona: ElsevierSaunders; 2013. p. 240-280.

9. Billing A, Frohlich D, Schildberg FW. Prediction of outcome Using the Mannheim peritonitis index in 2003 patients. British Journal of Surgery. 1994; 81(1):209-213.
10. Batra P, Gupta D, Batra R, Kothari R, Deshmukh P. Mannheim peritonitis Index as an evaluative tool in predicting mortality in patients of perforation peritonitis. CIBTech Journal of Surgery. 2013; 2(3): 30-36.

11. Linder Mm, Wacha H, Feldmann U, Wesch G, Streifensand R, Gundlach E. The Mannheim peritonitis index. an instrument for the intraoperative prognosis of peritonitis. Chirurg. 1987; 58(2):84-92.

12. Notash AY, Salimi J, Rahimian H, Fesharaki Ms, Abbasi A. Evaluation of Mannheim peritonitis Index an multiple organ failure score in patients with peritonitis. Indian J Gastroenterol. 2005; 24(5): 197-200.

13. Sharma S, Singh S, Makkar N, Kumar A, Singh-Sandhu M. Assessment of severity of peritonitis using mannheim peritonitis index. Niger J Surg. 2016; 22(2): 118-122.

14. Budzyński P, Dworak J, Natkaniec M, et al . The usefulness of the Mannheim Peritonitis Index score in assessing the condition of patients treated for peritonitis. Pol Przegl Chir.. 2015; 87(6): 301-306.

15. Barrera R. Eficacia en la predicción de mortalidad postoperatoria de pacientes con sepsis Intrabdominal Grave: índice de peritonitis de Mannheim-Charlson vs Apache II. Tesis de Bachiller. Trujillo, Perú: Universidad Nacional de Trujillo; 2014. 58 pp.

16. Vintimilla A, Molina S, Merchán M. Validación del Índice pronóstico de Mannheim en pacientes con peritonitis secundaria. Revista médica HJCA. 2012; 4(1): 14-18.

17. Carrillo-Hidalgo J. Valor predictivo de mortalidad del índice de peritonitis de Mannheim en el Hospital Regional de Alta Especialidad de Veracruz. Tesis de Titulo Cirujano General. Veracruz, México: Universidad Veracruzana; $2012.32 \mathrm{pp}$.

18. Vasquez C. Valor pronóstico del índice de Mannheim para mortalidad en pacientes con peritonitis del Hospital Belén de Trujillo. Tesis de Especialista Cirugía General. Trujillo, Perú: Universidad Nacional de Trujillo; $2010.41 \mathrm{pp}$.

Recibido: 13/09/2018

Aceptado: 27/12/2018 